\title{
The increase of signal-to-noise ratio in the spatial separation of signals by the curvature of the wavefront
}

\author{
Aleksandr Okorochkov*, Bogdan Malyutin, and Gleb Khripkov \\ Don State Technical University, Institute of Service and Business (Branch) DSTU in Shakhty, \\ 346500 Shakhty Shevchenko 147, Russian Federation
}

\begin{abstract}
The article presents the results of the research of the dependence of the signal-to-noise ratio in the focal spot of the reflecting passive phased antenna array (PPAA), made of planar diffraction elements, on the orientation of the PPAA relative to the structure of the interference field, its dimensions and distance from PPAA plane to the focal spot. The results of the research show that the signal-to-noise ratio mainly depends on the horizontal size of the PPAA. The increase of the vertical size of the array leads to the fact that the focal distance corresponding to the maximum of the signal-to-noise ratio increases with the practically unchanged value of this maximum.
\end{abstract}

\section{Introduction}

In accordance with the constant increase in volumes and requirements for the quality of transmitted information, there is a shortage of frequency resources, that is, bandwidths for data transmission for ensuring the required reliability of receiving.

As noted in [1], it is possible to increase the transmission speed of information without expanding the frequency band by various methods, including through the organization of parallel communication channels in the same frequency band. The main requirement here is the possibility of separating the signals on the receiving side. One of the parameters, the possibility of using it to separate signals in communication systems is just beginning to be discussed, is the curvature of the wavefront of an electromagnetic wave carrier in the region of the receiving antenna system. Publications on the use of the curvature of the wavefront in communication systems with the aim of increasing the information transfer rate began to appear only recently $[2,3]$.

In this paper we research the dependence of the signal / interference ratio on the aperture of the receiving antenna from the parameters of the reflective PPAA. However, this article uses the generally accepted term "signal-to-noise ratio" (SNR), in which a signal is the useful signal, a noise is the interference signal without taking into account the receiver's internal noise. The SNR at the aperture of the receiving antenna is researched depending on such parameters of the PPAA as its orientation relative to the structure of the interference field in the receiving region, the sizes of the PPAA, and the distance from the aperture PPAA plane to the focus area of the useful signal field (the aperture area of the receiving antenna).

*Corresponding author: okorochkov_a@mail.ru 


\section{Spatial separation of two signals on wavefront curvature}

When two parallel communication channels are organized in the same frequency band, the task of separating the signals at the receiving side arises, provided that for two signals there is a difference in the curvature of the wavefronts, that is, in the receiving plane, these signals have a different phase distribution.

In the article [4] the amplitude-phase distributions of two signals in the receiving plane along the coordinate $\mathrm{X}$-axis were obtained (Fig. 1,2), which we will call the horizontal direction.

The $Y$-axis, located in the receiving plane perpendicular to the $X$-axis, will be referred to as the vertical direction. Also, we introduce the $Z$-axis, directed along the normal to the plane of the PPAA towards the transmitting antennas. The plane of the PPAA coincides with the coordinate $X Y$-plane. Studies in [4] were carried out for signals with a frequency of $15 \mathrm{GHz}$ (wavelength $\lambda=2 \mathrm{~cm}$ ), which were radiated by three mirror antennas located $10 \mathrm{~m}$ apart. The distance from the transmitting antennas to the receiving plane was $10 \mathrm{~km}$. In order to achieve in the receiving plane of amplitude and phase distributions of the interference fields of the signals presented in Fig. 1 and 2, one signal (solid line) was transmitted in-phase by three antennas, and the second (dashed line) - with a linear phase shift at the inputs of the transmitting antennas.

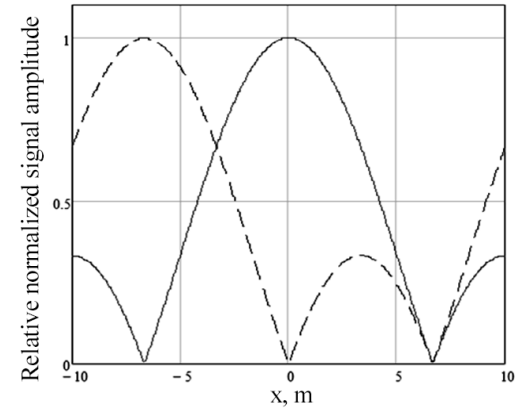

Fig. 1. The amplitude distribution in the plane of reception of the fields of two signals in the presence of a phase shift between them

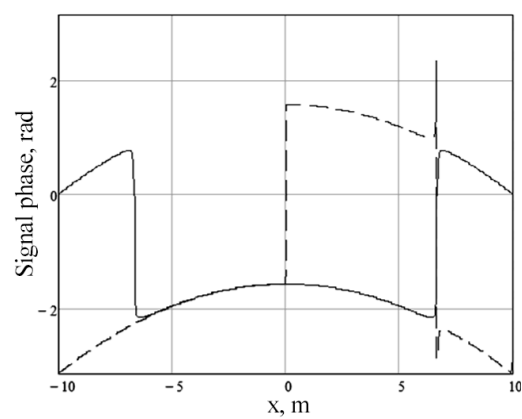

Fig. 2. The phase distribution in the receiving plane of the fields of two signals in the presence of a phase shift between them

As can be seen from Fig. 1 and 2, there are regions in the receiving plane where the amplitudes and phases of the two signals are very different. These areas can be used to accommodate PPAA. For example, if $x=0$, the amplitude of one signal (solid line) greatly exceeds the amplitude of other (dashed line), and the phase of the signal with a smaller amplitude at $x=0$ experiences a jump of $180^{\circ}$. Therefore, if the PPAA is located in the receiving area so that its center coincides with the point $\mathrm{x}=0$, and the diffraction elements thus provide after reflection such a phase distribution of the signal field with a greater amplitude (further signal) that it will focus on the aperture of the receiving antenna, then its level will significantly increase. A signal with a smaller amplitude (further noise) will be suppressed on the aperture of the receiving antenna due to the presence of antiphase components in it. Thus, the antiphase components of the noise field when reflected from the PPAA's elements located on different sides of its center along the horizontal direction will compensate each other and the noise will be strongly suppressed in the signal focusing area.

\section{Mathematical model of focusing PPAA}

To study the dependence of the SNR on the aperture of the receiving antenna on the parameters of the PPAA, a computer array model was used, created in the CST STUDIO 
SUITE Student Edition program. The PPAA model has a dimension of $5 \times 31$ elements (Fig. 3 ). The total number of elements of the PPAA was regulated by a reasonable time interval for the calculations, and the ratio of the number of elements along the sides of the rectangular opening of the array was determined by the tasks of the conducted studies. The distance between the elements of the array is equal to the wavelength $\lambda(2 \mathrm{~cm})$. The elements themselves have a square shape with side length $\lambda / 2$. In Fig. 4 PPAA elements are numbered in rows and columns.

In this paper, the SNR is the ratio of the power flows of the signal and noise fields through the aperture of the receiving antenna in the focus area of the useful signal field.

It should be noted that in the software modeling of PPAA, the amplitude and phase of the field emitted by one element were assumed constant over its entire surface.

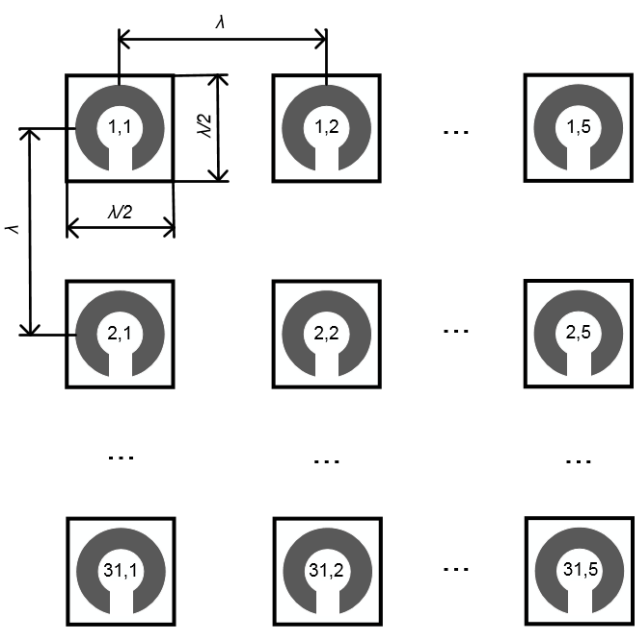

Fig. 3. Structure of the researched PPAA

This is a simplification of the computer model. In this case, it is permissible, because from the distribution of the amplitude and phase of the field along the horizontal axis in Fig. 1 and 2 it follows that on the length of the side of one element $(1 \mathrm{~cm})$ these quantities vary insignificantly.

\section{Research of dependence of SNR from spatial orientation of PPAA}

Let us research the distribution of the amplitudes of the focused electromagnetic fields of the signal and interference in the space between the plane of the PPAA and the focus point and find the SNR at the aperture of the receiving antenna in two cases: when the longer side of the array (Fig. 3) is oriented along the vertical direction, and when the longer side of the array is oriented along the horizontal direction.

Consider the first case. The distribution of the amplitude of the signal field in the $Y Z$ plane is shown in Fig. 4. The values on the coordinate axes are indicated in centimeters. The distance from the PPAA plane to the location of the receiving aperture (focusing region) was chosen arbitrarily and was $0.5 \mathrm{~m}$.

As can be seen from Fig. 4, the signal field "is compressed" in the YZ-plane in the region of the location of the receiving aperture, which leads to an increase in the signal amplitude. 
Let us research on the computer model of PPAA the behavior of the amplitude of the signal field in the region of the focal point. The distribution of the amplitude of the signal field along a line parallel to the $Y$-axis and located $0.5 \mathrm{~m}$ away from the PPAA plane is shown in Fig. 5. The distribution of the amplitude of the signal field along a line parallel to the $X$-axis and located $0.5 \mathrm{~m}$ away from the PPAA plane is shown in Fig. 6 .

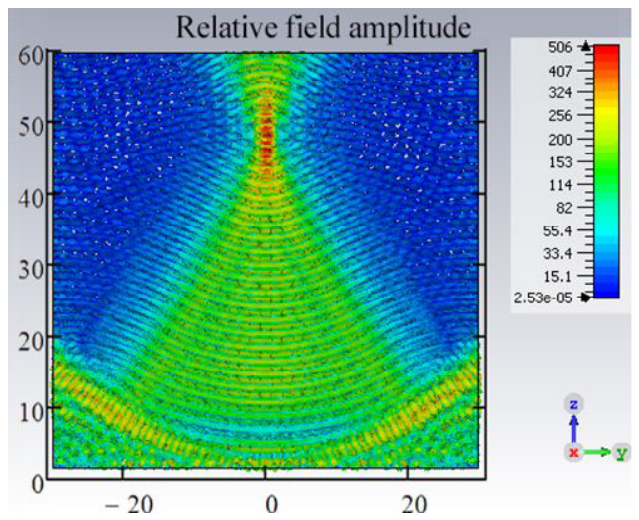

Fig. 4. Distribution of the amplitude of the signal field in the YZ-plane with the vertical orientation of the long side of the PPAA

On the last graphs, their maxima coincide with the point of focus lying on the optical axis of the PPAA, perpendicular to its opening at its center (at the point with the coordinates $x=0, y=0$ ).

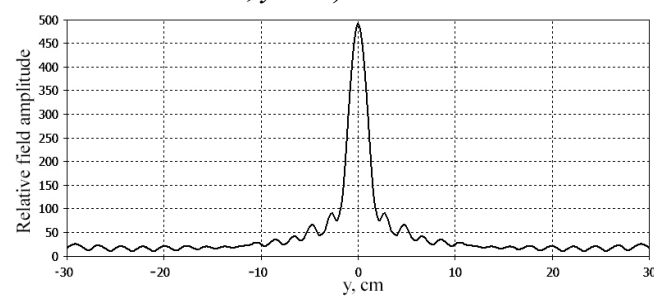

Fig. 5. The distribution of the signal field amplitude along the line passing parallel to the Yaxis through the focus, with the vertical orientation of the long side of the PPAA

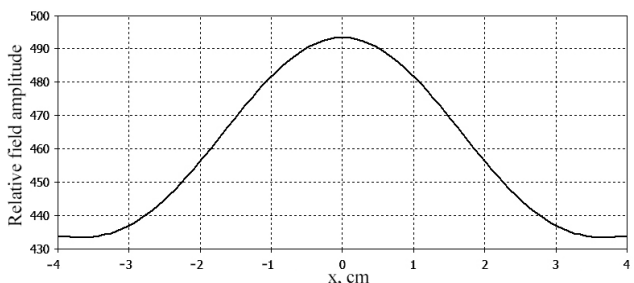

Fig. 6. The distribution of the signal field amplitude along the line passing parallel to the $\mathrm{X}$-axis through the focus, with the vertical orientation of the long side of the PPAA

In Fig. 5, we can see that in the vertical direction the amplitude peak of the signal is compressed more than in Fig. 6 in the horizontal direction, because along the $Y$-axis the PPAA is longer and contains more elements than along the $X$-axis. The dependence of the signal compression at the focus of the array on the length of its parties was already researched in [5].

Let us investigate the behavior of the amplitude of the noise field in the region of the focus point using the mathematical model of PPAA. The distribution of the amplitude of the noise field along a line parallel to the $Y$-axis and located $0.5 \mathrm{~m}$ away from the PPAA plane is shown in Fig. 7. The distribution of the amplitude of the noise field along a line parallel to the $X$-axis and located $0.5 \mathrm{~m}$ away from the PPAA plane is shown in Fig. 8.

In Fig. 7, it can be seen that the maximum of the amplitude of the noise field is in the vertical direction at the focus point of the signal. But its amplitude is much smaller than the amplitude of the signal at the same point, when compared with Fig. 5.

From the simulation results for the amplitude distribution of the noise field presented in Fig. 8, it can be concluded that in the horizontal direction in the focus area of the signal is 
the amplitude minimum noise. And it is evident that the amplitude distribution graph (Fig. 8 ) is asymmetric. This is due to the fact that the amplitude distribution of the noise field arriving at the PPAA is asymmetric with respect to its center (points with abscissa $\mathrm{x}=0$ in Fig. 1).

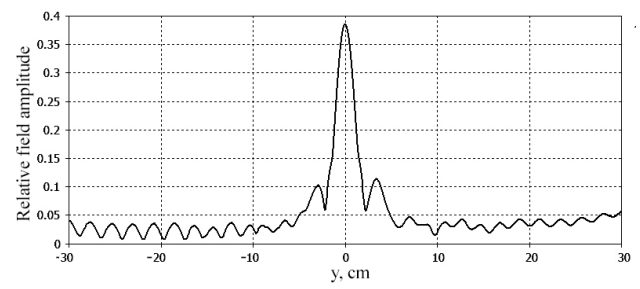

Fig. 7. The distribution of the amplitude of the noise field along a line passing parallel to the Yaxis through the focus, with the vertical orientation of the long side of the PPAA

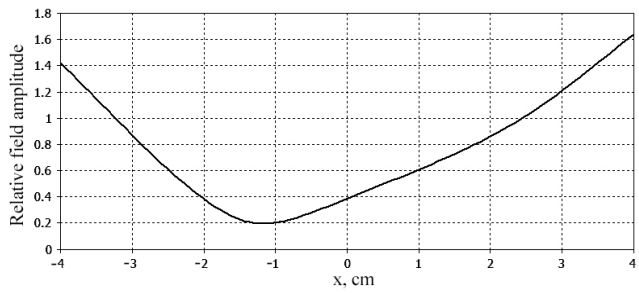

Fig. 8. The distribution of the amplitude of the noise field along a line passing parallel to the $\mathrm{X}$ axis through the focus, with the vertical orientation of the long side of the PPAA

The value of the SNR at the receiving antenna's aperture calculated in software modeling in the case where the longer side of the PPAA is located along the vertical direction is $61.7 \mathrm{~dB}$.

Let us consider the second variant of the PPAA location. When the long side of the PPAA is positioned along the horizontal direction, the distribution of the signal amplitude in the computational domain remains practically unchanged and will correspond to Fig. 4-6. This is due to the fact that the distribution of the amplitude of the signal field on the entire surface of the PPAA for any its orientation varies insignificantly because of the relatively small size of the PPAA (Fig. 1).

Let us investigate how the noise field behaves when reflected from the PPAA. The distribution of the amplitude of the noise field in the horizontal XZ-plane is shown in Fig. 9. In this figure, in the $X Z$-plane, a minimum of the noise amplitude is observed in the vicinity of the focus of the signal, since in this region two antiphase components of the noise field are added, strongly suppressing each other.

Let us investigate the behavior of the amplitude of the noise field in the region of the focus point of the signal in this case. The distribution of the amplitude of the noise field along the line parallel to the $X$-axis, and located $0.5 \mathrm{~m}$ from the plane of the PPAA, is shown in Fig. 10. The distribution of the amplitude of the noise field along a line parallel to the $Y$-axis, located $0.5 \mathrm{~m}$ away from the PPAA plane, is shown in Fig. 11.

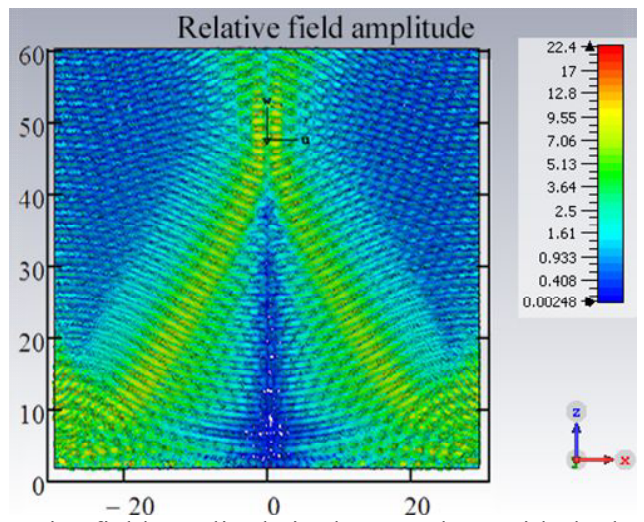

Fig. 9. Distribution of the noise field amplitude in the XZ-plane with the horizontal orientation of the long side of the PPAA 
From the graph in Fig. 10, it can be concluded that in the horizontal direction in the focus area of the signal is the amplitude minimum noise.

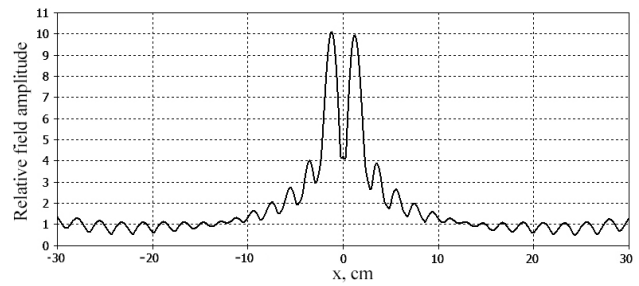

Fig. 10. The distribution of the amplitude of the noise field along a line passing parallel to the Xaxis through the focus, with the horizontal orientation of the long side of the PPAA

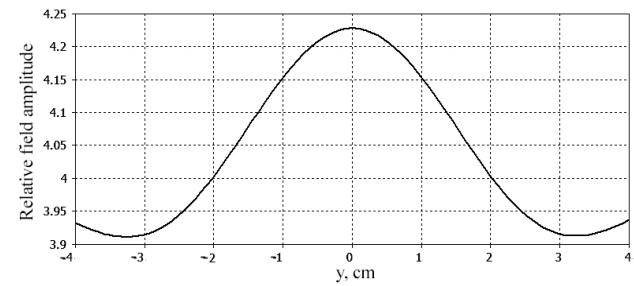

Fig. 11. Distribution of the amplitude of the noise field along a line passing parallel to the $\mathrm{Y}$ axis through the focus, with the horizontal orientation of the long side of the PPAA

The SNR calculated on the aperture of the receiving antenna for the case where the longer side of the PPAA is located along the horizontal direction was $36.5 \mathrm{~dB}$. Such a significant drop in the SNR is explained by the large influence in this case of the asymmetry of the amplitude behavior of the noise field in the vicinity of the zero amplitude point (Fig. 1). Therefore, when designing communication systems, the horizontal size of the PPAA and its position on the $X$-axis should be chosen based on the requirements for the minimum allowable SNR. At the same time, the loss of the power of the useful signal, if necessary, can be compensated by increasing the vertical size of the PPAA.

\section{Research of dependence of SNR from the position of the focus point and dimensions of PPAA}

Let us research the dependence of the SNR on the receiver aperture on the distance between the PPAA plane and the focal point for arrays of different sizes: 5x31 and 5x15 elements. As it was already explained, when the long side of the PPAA is oriented along the vertical direction, the SNR is higher than for the orientation along the horizontal direction. Therefore, the dependence of the SNR on the position of the focus point and the dimensions of the PPAA is calculated for the array whose long side is located vertically.

In Fig. 12 shows the dependence of the SNR on the distance between the plane of the array and the point of focus (focal distance) for the PPAA with the number of elements $5 \times 31$. $5 \times 15$.

In Fig. 13 shows an analogous dependence for the PPAA with the number of elements

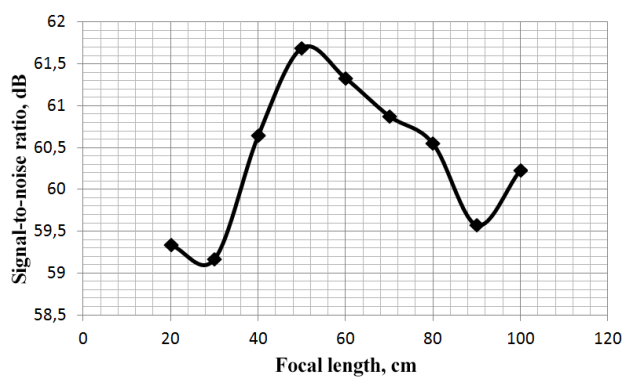

Fig. 12. Dependence of the SNR on the focal distance for the array with dimensions $5 \times 31$ element

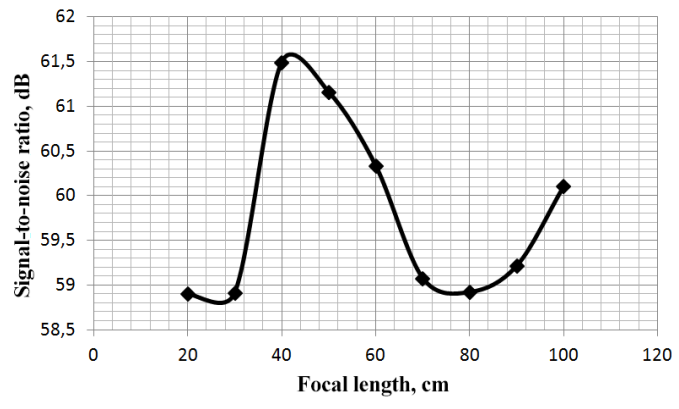

Fig. 13. Dependence of the SNR on the focal distance for the array with dimensions of $5 \times 15$ elements 
From the comparison of Fig. 12 and 13, two facts can be noted:

- with a decrease in the vertical dimension of the array, the focal point corresponding to the maximum SNR approaches the PPAA plane;

- the SNR is practically independent of the vertical array size, varying in the range $60 \pm$ $1.5 \mathrm{~dB}$.

The first fact should be considered when choosing the position of the aperture of the receiving antenna.

The second fact is explained by the fact that the structure of the interference field in the plane of the PPAA location is practically independent of the vertical coordinate y under the condition $y<<d$, where $d$ is the communication range. This again confirms the above conclusion about the dependence of the SNR only on the horizontal size of the PPAA.

\section{Conclusion}

Summarizing the results of the studies that led to the clarification of the dependence of the SNR on the orientation of the PFAR relative to the structure of the interference field in the reception region, the sizes of the PFAR and the focal distance, two conclusions can be drawn:

- the SNR mainly depends on the horizontal size of the PPAA due to the asymmetrical behavior of the amplitude of the noise field in the neighborhood of the point with zero amplitude and the violation of the strict antiphase of the noise field components at the PPAA plane;

- with an increase in the vertical size of the PPAA, the focal distance corresponding to the maximum of the SNR increases with the practically unchanged value of this maximum.

The received results of the carried out researches should be considered at designing of the receiving antenna modules (PPAA + receiving antenna) of systems of communication with spatial separation of signals by wavefront curvature.

\section{References}

1. B.B. Malyutin, A.I. Okorochkov, Nauchnaya vesna - 2017: Tekhnicheskie nayki [Scientific spring 2017: Technical science], 308 (2017) (in Russian)

2. E.A Yakornov, M.A. Kolomytsev, G.L. Avdeenko, O.Y. Lavrinenko, Visnik Natsional'nogo tekhnichnogo universitetu Ukraïni "KPI" Seriya - Radiotekhnika. Radioaparatobuduvannya [Bulletin of the National Technical University of Ukraine "KPI" Series - Radio Engineering. Radio equipment construction], 48, 97 (2012) (in Russian)

3. G.L. Avdeenko, E.A. Yakornov, Visnik Natsional'nogo tekhnichnogo universitetu Ukraïni "KPI" Seriya - Radiotekhnika. Radioaparatobuduvannya [Bulletin of the National Technical University of Ukraine "KPI" Series - Radio Engineering. Radio equipment construction], 52, 92 (2013) (in Russian)

4. G.L. Khripkov, A.I. Okorochkov, Nauchnaya vesna - 2017: Tekhnicheskie nayki [Scientific spring 2017: Technical science], 314 (2017) (in Russian)

5. A.S. Anikin, M.V. Krutikov, Doklady TUSUR [TUSUR reports], 4, 32 (2015) (in Russian) 